\title{
(2) Systematic benefit-risk assessment for ( OPEN ACCESS \\ buprenorphine implant: a semiquantitative method to support risk management
}

\section{Vicki Osborne (1) ,1,2 Miranda Davies,, Debabrata Roy, ${ }^{1,2}$ Francesco Tescione, ${ }^{3}$ Saad A W Shakir ${ }^{1,2}$}

10.1136/bmjebm-2019-111295

- Additional material is published online only. To view please visit the journal online (http://dx.doi.org/ 10.1136/bmjebm-2019111295).

${ }^{1}$ Drug Safety Research Unit, Southampton, UK ${ }^{2}$ School of Pharmacy and Biomedical Sciences, University of Portsmouth, Portsmouth, UK

${ }^{3}$ L Molteni \& C dei F.lli Alitti, Scandicci, Italy

Correspondence to: Dr Vicki Osborne, Drug Safety Research Unit, Southampton S031 1AA, UK; vicki. osborne@dsru.org
Check for updates

(- Author(s) (or their employer(s)) 2020. Re-use permitted under CC BY-NC. No commercial re-use. See rights and permissions. Published by BMJ.

To cite: Osborne $\mathrm{V}$,

Davies M, Roy D, et al. BMJ

Evidence-Based Medicine

2020;25:199-205.

\section{Abstract}

Background Prior to approval in the European Union, a systematic benefit-risk assessment was required to compare buprenorphine implant to sublingual buprenorphine as part of the license application to the European Medicines Agency.

Objective The Benefit-Risk Action Team framework was used to describe the overall benefitrisk of buprenorphine implant in comparison to sublingual buprenorphine.

Study selection/methods A value tree of key benefits and risks related to the implant formulation of buprenorphine was constructed. Risk differences (RD) or reporting ORs (ROR) and corresponding 95\% CIs were calculated for each outcome, along with the number needed to treat and number needed to harm. Swing weighting was assigned to outcomes and the weighted net clinical benefit (wNCB) was calculated. Findings Key benefits assessed: reduced risk of illicit opioid use ( $\mathrm{RD}=0.09,95 \% \mathrm{CI} 0.01$ to 0.17$)$, reduced risk of misuse and diversion $(\mathrm{ROR}=0.13$, 95\% CI 0.02 to 0.94), improved compliance and convenience $(\mathrm{RD}=0.20)$ and quality of life measures $(\mathrm{RD}=0.03)$. Key risks assessed: clinically significant implant breakage ( $\mathrm{RD}=0.01,95 \% \mathrm{CI} 0.00$ to 0.01 ), migration/missing implant $(\mathrm{RD}=0.01,95 \% \mathrm{CI} 0.00$ to 0.02), infection at insertion/removal site $(\mathrm{RD}=0.08$, $95 \% \mathrm{CI} 0.03$ to 0.12 ) and implant-related allergic reaction ( $\mathrm{RD}=0.07,95 \% \mathrm{CI} 0.03$ to 0.11 ). The wNCB for buprenorphine implant was 4.96 , which suggests a favourable benefit-risk profile.

Conclusions The benefit-risk profile of buprenorphine implant is considered favourable in comparison to sublingual buprenorphine, based on this semiquantitative analysis using available data. Further data from real-world use on benefits and risks should be used for ongoing monitoring of the benefit-risk profile of buprenorphine implants in the postmarketing setting.

\section{Introduction}

Opioid dependence is an important public health issue that, where left untreated, can result in debilitating health, social and economic consequences. ${ }^{12}$ While the opioid epidemic in the USA is well documented, ${ }^{3}{ }^{4}$ Europe also has an estimated 1.3 million high-risk opioid users. ${ }^{5}$ Treatment is vital to prevent adverse consequences and the standard approach is opioid substitution therapy with psychological and behavioural assistance. ${ }^{56}$

\section{Key messages}

\section{What is already known about this} subject?

> Treatment of opioid dependence is vital to prevent adverse consequences.

- The sublingual formulation of buprenorphine has been used in the treatment of opioid dependence for many years.

What are the new findings?

- The benefit-risk profile of buprenorphine implant is considered favourable in comparison to sublingual buprenorphine.

- The key benefits of improved compliance and convenience, reduced illicit opioid use and quality of life measures outweigh the key risks related specifically to the implant formulation.

\section{How might it impact on clinical practice in the foreseeable future? \\ - Physicians can make an informed decision on prescribing of the buprenorphine implant based on the benefit-risk profile.}

Buprenorphine is an opioid partial agonist/ antagonist which binds to the $\mu(\mathrm{mu})$ and $\kappa$ (kappa) receptors of the brain. Its affinity to reversibly bind to the $\mu$ receptors is the reason for its use in treatment of opioid dependence. In the European Union (EU), buprenorphine is available in several different formulations: sublingual tablets, subcutaneous injection and subcutaneous implant. ${ }^{7-9}$ The sublingual tablet formulation of buprenorphine has been available in the EU since the '90s, while the subcutaneous injection and subcutaneous implant formulations are new to the market. The sublingual tablets are administered under the supervision of a healthcare professional every 1-2 days, while the subcutaneous injection can be administered weekly or monthly. ${ }^{78}$ Buprenorphine subcutaneous implant is intended to provide continuous delivery of buprenorphine for 6 months and up to 1 year. After insertion of the implant, steady-state plasma buprenorphine concentration is usually achieved by 
week $4 .{ }^{9}$ Three double-blind phase III clinical trials were conducted to investigate the safety and efficacy of buprenorphine implant, including 309 patients aged 21-63 years with up to 6 months of follow-up. ${ }^{9}$

Buprenorphine implant is indicated for substitution treatment for opioid drug dependence in clinically stable adult patients who require no more than $8 \mathrm{mg} /$ day of sublingual buprenorphine, within a framework of medical, social and psychological treatment. ${ }^{9}$ It was initially approved by the Food and Drug Administration (FDA) in 2016 (under the brand name Probuphine) and subsequently by the European Medicines Agency (EMA) in 2019 (under the brand name Sixmo). ${ }^{10}{ }^{11}$ Prior to approval in the EU, a systematic benefitrisk assessment was required to compare buprenorphine implant to sublingual buprenorphine as part of the license application to the EMA. Consequently, this benefit-risk assessment was undertaken at the request of the marketing authorisation holder (MAH).

A systematic benefit-risk assessment is a useful tool to strengthen the ongoing monitoring of a medication's benefit-risk balance, while the use of a semiquantitative method standardises and supports the decision and communication of a benefit-risk assessment to regulatory authorities such as the EMA. ${ }^{12}$ We chose the Benefit-Risk Action Team (BRAT) framework for use in this assessment because it allows flexibility in choice of quantitative assessment, following the structured format of the qualitative framework. It was also specifically designed to assist communication with regulatory authorities. The framework design ensures that the decision-making process is transparent, while the quantitative component allows for any assumptions to be explored further by sensitivity analysis. ${ }^{13}$

This paper summarises the benefit-risk assessment undertaken for buprenorphine implant, prior to marketing authorisation in the EU.

\section{Objectives}

To examine the benefit-risk profile of buprenorphine implant compared with sublingual buprenorphine.

\section{Methods}

\section{Benefit-risk framework}

The BRAT framework was used to describe the overall benefit-risk of buprenorphine implant in comparison to sublingual buprenorphine. BRAT uses a six-step process to support the decision and communication of a benefit-risk assessment: define decision context, identify outcomes, identify data sources, customise framework, assess outcome importance, and display and interpret key benefit-risk metrics. ${ }^{12} 13$

\section{Population of interest}

For this assessment, the population of interest (the proposed target population for buprenorphine implant) was clinically stable adult patients who require no more than $8 \mathrm{mg} /$ day of sublingual buprenorphine, within a framework of medical, social and psychological treatment.

For the purposes of the benefit-risk assessment, the most appropriate comparator was considered to be sublingual buprenorphine since this is the treatment for which buprenorphine implant is proposed to substitute.

\section{Outcomes of interest}

Initially, all outcomes related to the buprenorphine implant, regardless of importance, were identified. Outcomes of interest were the important benefits and risks specific to the implant formulation, in comparison to the sublingual tablet formulation.
These consist of identified risks within the risk management plan (RMP), in addition to benefits perceived from the implant formulation of buprenorphine implant. Clinician judgement was used in this decision-making process, alongside consultation with experts in this area. A value tree was created to summarise the important benefits and risks driving the benefit-risk balance.

\section{Data sources and customisation of the framework}

We searched a variety of sources to identify suitable data for inclusion: PubMed, EMA website, FDA website and the FDA Adverse Event Reporting System (FAERS). In PubMed, we searched for papers on 'buprenorphine' and 'implant' $(\mathrm{n}=40)$, in addition to 'probuphine' $(\mathrm{n}=12)$. Additional filters were not applied due to the low number of results. The search strategy did not include 'Sixmo' as this brand name was not in use at the time of the search. Sublingual buprenorphine was the comparator of interest in all published studies. In addition, the RMP and Periodic Adverse Drug Experience Report for buprenorphine implant were obtained directly from the MAH.

Data were extracted from buprenorphine implant clinical trials (PRO-805, 806 and 814) ${ }^{14}$ and observational studies/data sets ${ }^{15-17}$ for each benefit and risk, for both buprenorphine implant and the comparator (sublingual buprenorphine).

In comparison to the sublingual formulation of buprenorphine, the key systemic adverse events and key systemic benefits reported with buprenorphine implant do not differ significantly because they are adverse events/benefits associated with the use of buprenorphine regardless of method of administration. Consequently, the key benefits and risks of interest with buprenorphine implant are those related to the implant formulation, which includes the risk of implant site reactions. The value tree was subsequently refined to reflect this, alongside the removal of benefits and risks for which insufficient data were available.

\section{Outcome assessment}

The number needed to treat (NNT) for each benefit and the number needed to harm (NNH) for each risk were calculated, where numerator and denominator data were available.

For each benefit, the NNT can be interpreted as the number of people who need to receive buprenorphine implant instead of sublingual buprenorphine in order for one person to benefit from buprenorphine implant treatment. As such, a lower NNT suggests a benefit occurs frequently with a small number of people treated. For each risk, the NNH can be interpreted as the number of people who need to receive buprenorphine implant instead of sublingual buprenorphine in order for one person to be affected by the risk of buprenorphine implant treatment. As such, a lower NNH suggests a risk occurs frequently with a small number of people treated.

The likelihood of being helped or harmed (LHH) was also calculated. ${ }^{18}$ The LHH is the ratio of the probability of benefit to the probability of harm and can be calculated as the $\mathrm{NNH}$ / NNT. Where the ratio is greater than 1, the benefit outweighs the risk. This analysis only uses crude data and so does not take into account the relative importance of each benefit and risk.

\section{Quantitative assessment}

Each benefit and risk was assigned a weight based on its perceived importance or severity by a clinician. Swing weighting was used to assign weights to each outcome. First, the key benefits and risks were ranked in order of importance. A score of 100 was assigned to the most important outcome and then subsequent outcomes were scored in reference to this, by considering the importance 


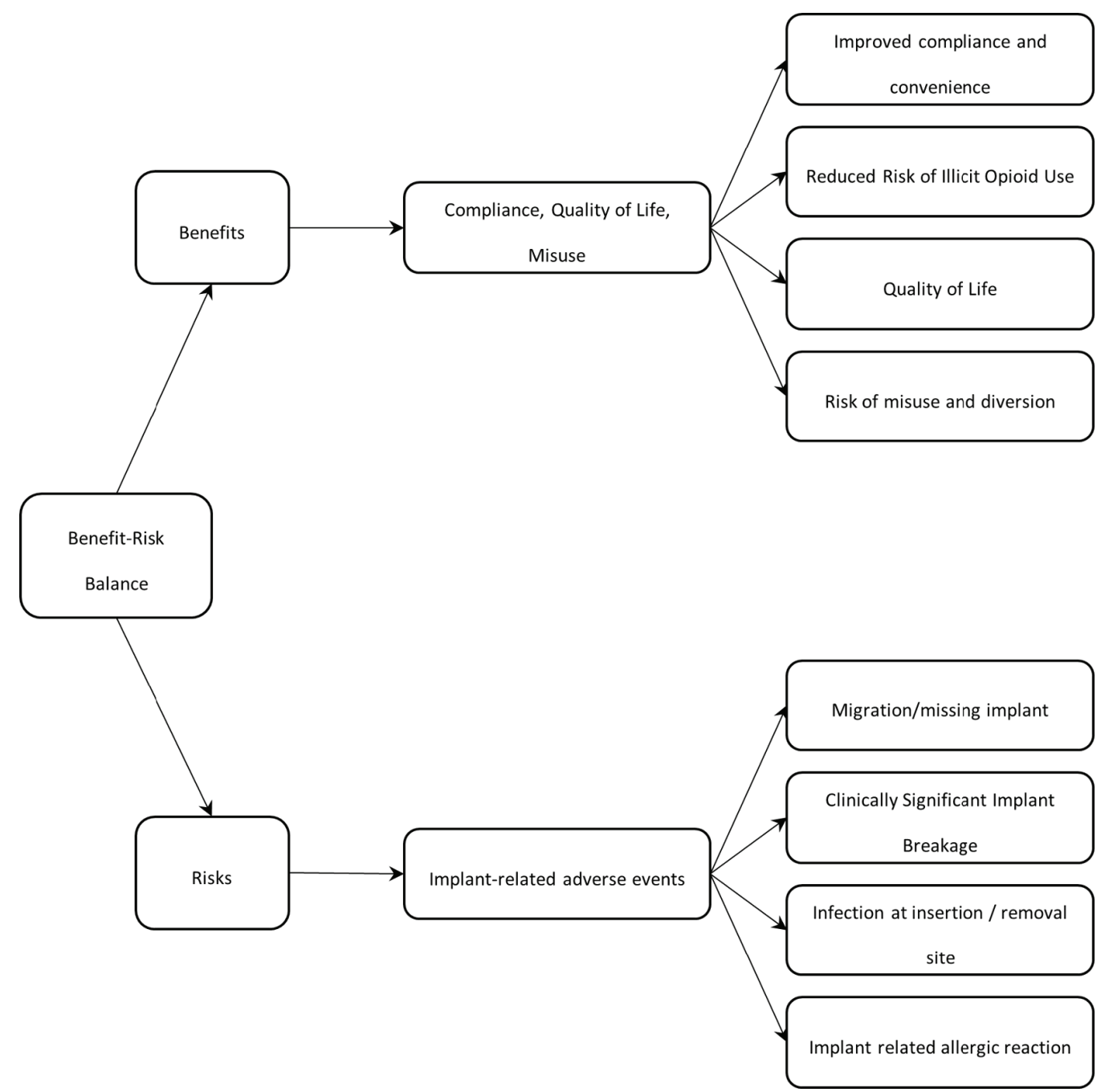

Figure 1 Value tree of key benefits and risks identified for buprenorphine implant.

of each outcome in relation to the most important outcome. The weights for each outcome were then normalised to add to 100 . The weighted net clinical benefit (wNCB) was subsequently calculated using these weights. ${ }^{13} 1920$ The method of Sutton et al was used, where benefits of buprenorphine implant versus sublingual buprenorphine have a positive contribution to the wNCB and risks have a negative contribution. ${ }^{20}$ The formula used for calculation of the wNCB is provided in online supplementary material. The overall wNCB is positive (benefit outweighs the risk) where wNCB is $>0$.

A sensitivity analysis was undertaken to determine the robustness of the weights and whether significant changes would alter the benefit-risk profile for buprenorphine implant. Three scenarios were examined where the benefit weights were reduced by a third, a half and two-thirds, with the risk weights increasing in equal proportions (see online supplementary material).

\section{Results}

The value tree reflecting the key benefits and risks related specifically to the implant formulation is displayed in figure 1. Data for these outcomes for both buprenorphine implant and the comparator group of sublingual buprenorphine, and the key benefit-risk summary table are displayed in online supplementary material.

\section{Benefits}

\section{Reduced risk of illicit opioid use}

In study PRO-814, which compared buprenorphine implant $(n=84)$ to sublingual buprenorphine $(n=89)$, the primary efficacy outcome was no more than 2 out of 6 months where there was any evidence of illicit opioid use. A higher percentage of those in the buprenorphine implant group had no more than 2 out of 6 months where there was any evidence of illicit opioid use (96\%) compared with those on sublingual buprenorphine (88\%; risk difference $(\mathrm{RD})=0.09,95 \% \mathrm{CI} 0.01$ to 0.17 ).

\section{Risk of misuse and diversion}

The FAERS contains spontaneous reports for buprenorphine implant and sublingual buprenorphine, as used in real life in the general population in the USA. ${ }^{16}$ The total database to 30 September 2018 contained 72 adverse event reports for buprenorphine implant (Probuphine) and 3852 adverse event reports for sublingual buprenorphine (Subutex). Of these adverse event reports, there was one report of intentional product misuse for buprenorphine implant and 375 reports of intentional product misuse for Subutex. This equates to a reporting OR (ROR) of 0.13 ( $95 \%$ CI 0.02 to 0.94 ), suggesting a reduction in risk of misuse with buprenorphine implant compared with sublingual buprenorphine. 
Drug diversion was not reported for buprenorphine implant in FAERS, but there were 49 adverse event reports of drug diversion with sublingual buprenorphine.

\section{Improved compliance and convenience}

Retention in treatment was considered a surrogate marker of compliance. A cost-effectiveness analysis undertaken using a Markov model populated with relevant inputs from peer-reviewed literature examined retention in treatment for buprenorphine implant compared with sublingual buprenorphine. ${ }^{15}$ Inputs were drawn from the peer-reviewed literature because clinical trialbased evidence for retention in treatment would not have reflected the real-world perspective due to the artificial nature of follow-up and application of retention methods employed in clinical trials. Over a 12-month modelled time horizon, the buprenorphine implant cohort achieved higher rates of retention in treatment (78\% vs 58\%; $\mathrm{RD}=0.20$ ) compared with sublingual buprenorphine, suggesting improved compliance with buprenorphine implant compared with sublingual buprenorphine.

\section{Quality of life measures}

The cost-effectiveness analysis undertaken using a Markov model populated with relevant inputs from peer-reviewed literature also examined quality of life measures for buprenorphine implant compared with sublingual buprenorphine. ${ }^{15}$ Quality-adjusted life years (QALY) were calculated and over a 12-month modelled time horizon, the buprenorphine implant cohort achieved more QALYs compared with sublingual buprenorphine (0.83 vs 0.80 ), suggesting better quality of life with buprenorphine implant compared with sublingual buprenorphine.

\section{Risks}

\section{Infection at insertion/removal site}

Only studies that used the licensed, current insertion technique were included when considering this outcome; this represents the approved insertion technique which should be used by physicians in the real-world setting. Two clinical trials used the current technique for insertion and removal of buprenorphine implant; PRO-806 and PRO-814 ( $\mathrm{n}=201$ buprenorphine implant, $\mathrm{n}=89$ sublingual buprenorphine). The percentage of patients who experienced infection at the insertion/removal site was $9 \%$ in the buprenorphine implant group, compared with $1 \%$ in the sublingual buprenorphine group who had a placebo implant inserted $(\mathrm{RD}=0.08,95 \% \mathrm{CI} 0.03$ to 0.12 ). It is acknowledged that the use of placebo implants does not apply in the real-world setting, so we also examined the RD if incidence was $0 \%$ in the sublingual buprenorphine group and this did not impact the conclusions drawn.

\section{Migration/missing implant}

No cases of distant migration have been reported with buprenorphine implant. Only studies that used the licensed, current insertion technique were included when considering this outcome. In the two clinical trials which used the current technique for insertion and removal of buprenorphine implant, two patients (1\%; $\mathrm{RD}=0.01,95 \% \mathrm{CI} 0.00$ to 0.02 ) had implants or fragments that could not be located in the buprenorphine implant group and were therefore not removed at the end of the study.

\section{Implant-related allergic reaction}

Only studies that used the licensed, current insertion technique were included when considering this outcome. In the two clinical trials which used the current technique for insertion and removal of buprenorphine implant, the percentage of patients who experienced implant site pruritus, erythema and oedema was 4\%, 2\% and $1 \%$, respectively. The overall percentage of patients who experienced these implant-related allergic reactions was $8 \%$, while the overall percentage in the sublingual buprenorphine group who had a placebo implant was $1 \%(\mathrm{RD}=0.07,95 \% \mathrm{CI} 0.03$ to 0.11$)$.

\section{Clinically significant implant breakage}

Implant breakage is likely to only be of concern where clinically significant and perceived to be a safety concern to the patient. For example, where broken implants could be removed without problems, these are not considered to be clinically significant. Clinically significant breakage would result in incomplete removal or difficulty with removal. In addition, the surface area of the implant is minimally affected by mechanical stress; breakage into two would increase the surface area by only $4.6 \%$ and should not result in dose dumping or any discernible effect on drug release. ${ }^{14}$ Only studies that used the current insertion technique were included when considering this outcome. In the two clinical trials which used the current technique for insertion and removal of buprenorphine implant ( $\mathrm{n}=201$ buprenorphine implant) and from postmarketing experience $(\mathrm{n}=835)$, the percentage of patients who experienced clinically significant implant breakage was $1 \%$.

\section{NNTs and NNHs}

The crude number NNT for each key benefit, alongside the crude number NNH for each key risk, is displayed in table 1. Based on the NNTs, five people would need to receive buprenorphine implant in order for one person to benefit from improved compliance and convenience.

Based on the NNHs, 200 people would need to receive buprenorphine implant in order for one person to experience a clinically significant implant breakage.

Table 2 indicates a positive LHH ratio for improved compliance and convenience and for reduced risk of illicit opioid use when examining all harms. Quality of life measures had a positive LHH ratio for two of the key risks identified with buprenorphine implant migration/missing implant and clinically significant implant breakage. The LHH is not positive for quality of life measures for the risks of infection at insertion/removal site and implant-related allergic reaction. However, this method does not account for the importance of each benefit and risk examined and does not examine the complete benefit-risk profile for buprenorphine implant while taking this into account.

\section{Weighted net clinical benefit}

The swing weights assigned to outcomes by a clinician are presented in online supplementary material, with the swing weights normalised to sum to 100 while maintaining the ratio between them. Online supplementary material displays the results of the wNCB analysis. The overall wNCB for buprenorphine implant is 4.96 , which suggests a favourable benefit-risk profile.

\section{Discussion}

Four key benefits for buprenorphine implant were identified during this assessment which relate specifically to the long-acting, extended release implant formulation: improved convenience and compliance, reduced risk of illicit opioid use, reduced risk of misuse and diversion and quality of life measures. Conversely, four key risks were also identified for buprenorphine implant: migration/ missing implant, clinically significant implant breakage, infection at insertion/removal site and implant-related allergic reaction. 


\begin{tabular}{|c|c|c|c|}
\hline Outcome name & Number needed to treat (NNT) & Number needed to harm (NNH) & $95 \% \mathrm{Cl}$ \\
\hline \multicolumn{4}{|l|}{ Benefits } \\
\hline Improved compliance and convenience & 5.00 & & Not calculable* \\
\hline Reduced risk of illicit opioid use & 11.38 & & 5.99 to 113.64 \\
\hline Quality of life measures & 32.26 & & Not calculable* \\
\hline Risk of misuse and diversion & Not calculablet & Not calculablet & Not calculable \\
\hline \multicolumn{4}{|l|}{ Risks } \\
\hline Migration/missing implant & & 100.00 & -250.00 to 41.67 \\
\hline Clinically significant implant breakage & & 200.00 & 111.11 to 1000.00 \\
\hline Infection at insertion/removal site & & 12.77 & 8.13 to 30.21 \\
\hline Implant-related allergic reaction & & 14.62 & 8.93 to 40.00 \\
\hline
\end{tabular}

*Data not available to calculate $95 \% \mathrm{Cl}$.

tSpontaneous reporting data only; data not available to calculate NNT or NNH.

RDs and RORs revealed significant point estimates for two benefits (reduced risk of illicit opioid use and reduced risk of misuse and diversion). The same was observed for the risks of infection at insertion/removal site and implant-related allergic reaction.

In clinical trial PR0-814, a higher percentage of those in the buprenorphine implant group had no more than 2 out of 6 months where there was any evidence of illicit opioid use compared with those on sublingual buprenorphine. ${ }^{21}$ Data from this study are likely to be most relevant to the proposed indication group, since the population studied were clinically stable subjects on buprenorphine maintenance therapy. This study also only required a small proportion of patients in the buprenorphine implant group to receive additional sublingual buprenorphine, suggesting clinical stability on treatment which was comparable with the sublingual buprenorphine group and supporting the conclusion that results from study PRO-814 on reduced risk of illicit opioid use are likely to be generalisable to the indicated real-world population.

A reduction in risk of misuse with buprenorphine implant compared with sublingual buprenorphine was observed. Misuse of

\begin{tabular}{ll}
$\begin{array}{l}\text { Table } 2 \text { Likelihood of being helped or harmed for buprenorphine } \\
\text { implant }\end{array}$ & $\begin{array}{l}\text { Likelihood of being } \\
\text { helped or harmed } \\
\left(\mathrm{LHH}^{*}\right)\end{array}$ \\
\hline & 20.00 \\
Outcome & 40.00 \\
\hline Improved compliance and convenience & 2.55 \\
\hline Migration/missing implant & 2.92 \\
\hline Clinically significant implant breakage & \\
\hline Infection at insertion/removal site & 8.79 \\
\hline Implant-related allergic reaction & 17.57 \\
\hline Reduced risk of illicit opioid use & 1.12 \\
\hline Migration/missing implant & 1.28 \\
\hline Clinically significant implant breakage & \\
\hline Infection at insertion/removal site & 3.10 \\
\hline Implant-related allergic reaction & 6.20 \\
\hline Quality of life measures & 0.40 \\
\hline Migration/missing implant & 0.45 \\
\hline Clinically significant implant breakage & \\
\hline Infection at insertion/removal site & Implant-related allergic reaction
\end{tabular}

*Calculated as number needed to harm/number needed to treat (NNH/ NNT). the sublingual formulation has been studied previously and can involve altering the intended route of administration by injecting buprenorphine $e^{22-24}$ or using buprenorphine intranasally. ${ }^{2526}$ The buprenorphine implant has been formulated to be abuse and diversion deterrent. The difficulty in misusing the implant versus the sublingual formulation may account for the reduction in risk of misuse; however, further studies in the real-world setting will provide more evidence to support this conclusion.

Data on drug diversion were not available for the buprenorphine implant. Diversion of the sublingual formulation has been observed previously in other studies. ${ }^{27-29}$ The subcutaneous placement of the implant makes diversion unlikely. Further studies in the real-world setting are needed in future to confirm this assumption. In terms of additional sublingual buprenorphine which may be given alongside the buprenorphine implant, potential drug diversion could also be prevented. Specifically, in patients with milder opioid addiction, who are relatively clinically stable, minimal additional sublingual buprenorphine to reduce opioid cravings is required. This would result in less sublingual tablets to divert or misuse.

Risk of infection at insertion/removal site and implant-related allergic reactions are risks specific to the implant formulation of buprenorphine. Clinical trial data allowed us to compare this risk with sublingual buprenorphine due to the use of placebo implants, though it is acknowledged that these would not be used in the real-world setting. However, the risk of all implant-related adverse events was low, especially for serious adverse events such as clinically significant implant breakage and missing or migrated implant, and we did not find that these risks outweighed the benefits of the buprenorphine implant.

Overall, the wNCB for buprenorphine implant was 4.96, which suggests a favourable benefit-risk profile. In addition, the results from the sensitivity analysis (see online supplementary material) suggest that our swing weights would have to change significantly for the benefits and risks to result in an unfavourable benefit-risk profile and show the robustness of the benefit-risk profile across a range of assumptions. These findings are applicable to clinically stable adult patients only.

\section{Strengths and limitations}

The crude analyses using NNT and NNH should be interpreted with caution because they do not account for the importance of each benefit and risk. A wNCB analysis was undertaken to address this limitation. 
The swing weights applied were based on clinician judgement and are subjective. They may not reflect patient preference or consideration of importance. However, a sensitivity analysis was undertaken to determine the robustness of these weights and it would take a significant change in the weights to result in an unfavourable benefit-risk profile.

Data from clinical trials were used for some of the benefits and risks assessed. RDs identified from clinical trial data may not be reflective of the RD in real-world use. However, the sensitivity analysis examined the robustness of the benefit-risk profile across a range of assumptions, to ensure the benefit-risk profile was not altered by minor changes.

Data from one study for two of the benefit outcomes (improved compliance and convenience and quality of life measures) were obtained from modelled data and so may not reflect real-world benefit. However, inputs were drawn from the peer-reviewed literature, reflecting the real-world perspective.

Sample sizes for each outcome were limited to those available in the original studies and may not have adequate power to detect differences in risk between the two treatment groups, especially where the outcomes examined were not the primary outcome of interest. However, this assessment was limited to data available at the time. Ongoing monitoring and review of the benefits and risks via the Periodic Benefit-Risk Evaluation Report will allow for any changes identified in the postmarketing setting to be incorporated into the framework used in this assessment.

\section{Conclusions}

Overall, the benefit-risk profile of buprenorphine implant is considered favourable in comparison to sublingual buprenorphine, based on this semiquantitative analysis using available data. The key benefits of improved compliance and convenience, reduced illicit opioid use and quality of life measures outweigh the key risks related specifically to the implant formulation. Further data from real-world use on benefits and risks should be used for ongoing monitoring of the benefit-risk profile of buprenorphine implants in the postmarketing setting.

Contributors VO, MD, DR and SAWS designed the study and consulted with FT regarding the design. VO, MD and DR conducted the study and interpreted the data. The manuscript was written primarily by VO. All authors reviewed, contributed to revisions and approved the manuscript, and accept full responsibility for its overall content.

Funding The Drug Safety Research Unit (DSRU) is an independent academic institution which works in association with the University of Portsmouth. The DSRU has received funding from L Molteni \& C dei F.lli Alitti, the marketing authorisation holder for Sixmo, to conduct this study. The funding source was consulted during the study design phase but the final decision on study design was made by the DSRU.

Disclaimer The funding source had no role in the collection, analysis and interpretation of data, though was consulted at the time of writing the report and the manuscript. The final decision on content and to submit the article for publication was made by the DSRU.

Competing interests FT is an employee of L Molteni \&t C dei F.lli Alitti, the marketing authorisation holder for Sixmo. VO, MD, DR and SAWS have no competing interests to declare.

Patient consent for publication Not required.
Provenance and peer review Not commissioned; externally peer reviewed.

Data availability statement Data sharing is not applicable as no data sets were generated and/or analysed for this study. Data used in this analysis are available from the references supplied.

Open access This is an open access article distributed in accordance with the Creative Commons Attribution Non Commercial (CC BY-NC 4.0) license, which permits others to distribute, remix, adapt, build upon this work non-commercially, and license their derivative works on different terms, provided the original work is properly cited, appropriate credit is given, any changes made indicated, and the use is non-commercial. See: http://creativecommons.org/licenses/by-nc/4.0/.

\section{ORCID iD}

Vicki Osborne http://orcid.org/0000-0002-3669-6084

\section{References}

1 Degenhardt L, Charlson F, Mathers B, et al. The global epidemiology and burden of opioid dependence: results from the global burden of disease 2010 study. Addiction 2014;109:1320-33.

2 Hser Y-I, Evans E, Grella C, et al. Long-Term course of opioid addiction. Harv Rev Psychiatry 2015;23:76-89.

3 Centers for Disease Control and Prevention. Drug overdose deaths, 2018. Available: https://www.cdc.gov/drugoverdose/data/statedeaths.html [Accessed 16 Aug 2019].

4 Scholl L. Drug and Opioid-Involved overdose deaths - United States, 2013-2017. MMWR Morb Mortal Wkly Rep 2019;67.

5 European Monitoring Centre for Drugs and Drug Addiction. European drug report, 2019. Available: http://www.emcdda.europa.eu/system/files/ publications/11364/20191724_TDAT19001ENN_PDF.pdf

6 World Health Organization. Guidelines for the Psychosocially Assisted Pharmacological Treatment of Opioid Dependence. Geneva: World Health Organization, 2009. Available: http://www.ncbi.nlm.nih.gov/books/ NBK143185/ [Accessed 16 Aug 2019].

7 European Medicines Agency. Suboxone. Eur. Med. Agency, 2018. Available: https://www.ema.europa.eu/en/medicines/human/EPAR/ suboxone [Accessed 16 Aug 2019].

8 European Medicines Agency. Buvidal. Eur. Med. Agency, 2018. Available: https://www.ema.europa.eu/en/medicines/human/EPAR/buvidal [Accessed 16 Aug 2019].

9 L. Molteni \&t C. dei F.lli Alitti Soc.Es.S.p.A. Summary of Product Characteristics: Sixmo, 2019. Available: https://www.ema.europa.eu/en/ documents/product-information/sixmo-epar-product-information_en.pdf

10 European Medicines Agency. New long-lasting implant to treat opioid dependence. Eur. Med. agency, 2019. Available: https://www.ema.europa. eu/en/news/new-long-lasting-implant-treat-opioid-dependence [Accessed 16 Aug 2019].

11 U.S. Food and Drug Administration. FDA approves first buprenorphine implant for treatment of opioid dependence. FDA, 2016. Available: http:// www.fda.gov/news-events/press-announcements/fda-approves-firstbuprenorphine-implant-treatment-opioid-dependence [Accessed 16 Aug 2019].

12 PROTECT. PROTECT benefit-risk, 2015. Available: http://protectbenefitrisk. eu/ [Accessed 11 Jan 2019].

13 Nixon R, Dierig C, Mt-Isa S, et al. A case study using the PrOACT-URL and BRAT frameworks for structured benefit risk assessment. Biom J 2016;58:8-27.

14 European Medicines Agency. Sixmo: assessment report, 2019. Available: https://www.ema.europa.eu/en/documents/assessment-report/sixmo-eparpublic-assessment-report_en.pdf

15 Carter JA, Dammerman R, Frost M. Cost-effectiveness of subdermal implantable buprenorphine versus sublingual buprenorphine to treat opioid use disorder. J Med Econ 2017;20:893-901.

16 Center for Drug Evaluation and Research. FDA Adverse Event Reporting System (FAERS) - FDA Adverse Event Reporting System (FAERS) Public Dashboard, 2019. Available: https://www.fda.gov/drugs/guidancecomplian 
ceregulatoryinformation/surveillance/adversedrugeffects/ucm070093.htm [Accessed 4 Jan 2019].

17 Titan pharmaceuticals. Probuphine periodic adverse drug experience report 5/27/2018 to 8/26/2018 2018 .

18 Andrade C. Likelihood of being helped or Harmed as a measure of clinical outcomes in psychopharmacology. J Clin Psychiatry 2017;78:e73-5.

19 Holden WL, Juhaeri J, Dai W. Benefit-risk analysis: a proposal using quantitative methods. Pharmacoepidemiol Drug Saf 2003;12:611-6.

20 Sutton AJ, Cooper NJ, Abrams KR, et al. A Bayesian approach to evaluating net clinical benefit allowed for parameter uncertainty. J Clin Epidemiol 2005;58:26-40.

21 Rosenthal RN, Lofwall MR, Kim S, et al. Effect of buprenorphine implants on illicit opioid use among abstinent adults with opioid dependence treated with sublingual buprenorphine: a randomized clinical trial. JAMA 2016;316:282-90.

22 Comer SD, Sullivan MA, Vosburg SK, et al. Abuse liability of intravenous buprenorphine/naloxone and buprenorphine alone in buprenorphinemaintained intravenous heroin abusers. Addiction 2010;105:709-18.

23 Moratti E, Kashanpour H, Lombardelli T, et al. Intravenous misuse of buprenorphine: characteristics and extent among patients undergoing drug maintenance therapy. Clin Drug Investig 2010;30:3-11.
24 Vidal-Trecan G, Varescon I, Nabet N, et al. Intravenous use of prescribed sublingual buprenorphine tablets by drug users receiving maintenance therapy in France. Drug Alcohol Depend 2003;69:175-81.

25 Hakansson A, Medvedeo A, Andersson M, et al. Buprenorphine misuse among heroin and amphetamine users in Malmo, Sweden: purpose of misuse and route of administration. Eur Addict Res 2007;13:207-15.

26 Middleton LS, Nuzzo PA, Lofwall MR, et al. The pharmacodynamic and pharmacokinetic profile of intranasal crushed buprenorphine and buprenorphine/naloxone tablets in opioid abusers. Addict Abingdon Engl 2011;106:1460-73.

27 Lavonas EJ, Severtson SG, Martinez EM, et al. Abuse and diversion of buprenorphine sublingual tablets and film. J Subst Abuse Treat 2014;47:27-34.

28 Lofwall MR, Walsh SL. A review of buprenorphine diversion and misuse: the current evidence base and experiences from around the world. $J$ Addict Med 2014;8:315-26.

29 Winstock AR, Lea T, Sheridan J. Prevalence of diversion and injection of methadone and buprenorphine among clients receiving opioid treatment at community pharmacies in New South Wales, Australia. Int J Drug Policy 2008;19:450-8. 